Proceedings of the International School and Conference on Optics and Optical Materials, ISCOM07, Belgrade, Serbia, September 3-7, 2007

\title{
Group Delay in Semiconductor Structures with Energy Dependent Effective Mass
}

\author{
S. KOČINAC \\ Faculty of Technology, Dep. of Physics, Karnegijeva 4, 11120 Belgrade, Serbia \\ AND V. Milanović \\ Faculty of Electrical Engineering \\ Bulevar kralja Aleksandra 73, 11120 Belgrade, Serbia

\begin{abstract}
We investigate tunneling times of a particle with energy dependent effective mass for a one-dimensional real potential. General relations between phase, group and dwell times are obtained for a desired potential shape. For the textbook case of a real rectangular potential barrier the explicit relations for relevant times are derived, which reveal that the nonparabolicity, depending on the energy of incident particle, may substantially increase the group time in realistic structures. Further, we extend this theory to the case of absorptive media described by complex potentials, via introduction of a new absorptive tunneling time $\tau_{\mathrm{a}}$. Depending on whether the short wavelength or long wavelength limit is considered, maximization of $\tau_{\mathrm{a}}$ results in a very different shape of a complex rectangular potential.
\end{abstract}

PACS numbers: 03.65.Xp, 73.21.Fg, 73.40.Gk, 73.43.Jn

\section{Introduction}

The old problem of adequately defining relevant tunneling times regained on its urgency after realization of transistors operating on tunneling through double potential barrier by Capasso et al. [1]. The fact that the tunneling time is considered to be one of the most important parameters in the evaluation of high-speed device performance, gave rise in last years to the increasing interest in spin-dependent tunneling in the field of spintronics [2]. A variety of answers to the question of how much time does the tunneling take has been offered in the last six decades [3-9].

Numerous definitions of the tunneling time can be found in [10], and of prime importance are the so-called phase time or group delay time and dwell time. The 
two contributions of asymptotic phase times seemed impossible to be disentangled, until a satisfactory answer was given in [11, 12]. In [13] Winful showed that the group delay is equal to the sum of the dwell time and self-interference delay.

In the first section of the paper we extend Winful's theory to the case of energy dependent effective mass, including the terms due to nonparabolicity, and derive the explicit relations for the tunneling times for the text book case of rectangular real barriers. The second part of the paper deals with the relationship between group, dwell, and self-interference time for a complex potential of an arbitrary shape.

\section{Real potential}

We start with one-dimensional real potential barrier $V(z), 0<z<L$. A particle of momentum $\hbar k$ and energy $E$ propagates from left to right. Upon reaching $z=0$ a part of wave is reflected and the rest transmitted. Transmission and reflection probabilities are given by the squares of amplitudes of transmission coefficient $T=|T| \exp \left(\mathrm{i} \phi_{\mathrm{t}}\right)$ and reflection coefficient $R=|R| \exp \left(\mathrm{i} \phi_{\mathrm{r}}\right)$, respectively. The total group delay (the delay in the appearance of the wave packet at the front and at the end of the potential barrier) is given by the sum of the group delay in transmission $\tau_{\mathrm{gt}}$ and reflection: $\tau_{\mathrm{g}}=|T|^{2} \hbar \mathrm{d} \phi_{0} / \mathrm{d} E+|R|^{2} \hbar \mathrm{d} \phi_{\mathrm{r}} / \mathrm{d} E$, where $\phi_{0}=\phi_{\mathrm{t}}+k L$.

On the other hand, the dwell time is the total time the particle spends in the barrier, regardless of whether it is mostly transmitted or reflected $[5,14]$ :

$$
\tau_{\mathrm{d}}=\frac{m}{\hbar k} \int_{0}^{L}|\psi(z)|^{2} \mathrm{~d} z
$$

To establish the relationship between group and dwell times given we use the procedure given in [14]. The general expression for the wave function in case of finite barrier is

$$
\begin{aligned}
& \psi_{\mathrm{L}}=\mathrm{e}^{\mathrm{i} k z}+R \mathrm{e}^{-\mathrm{i} k z}, \quad-\infty<z \leq 0, \\
& \psi_{\mathrm{B}}=\chi(z, k), \quad 0 \leq z \leq L, \\
& \psi_{\mathrm{R}}=T \mathrm{e}^{\mathrm{i} k z}, \quad L \leq z<+\infty,
\end{aligned}
$$

where indices $\mathrm{L}, \mathrm{B}$, and $\mathrm{R}$ denote left, barrier, and right part of the $z$ axis and $k^{2}=2 m_{0}(E) E / \hbar^{2}$. The nonparabolicity is accounted for via $m(E)=m^{*}[1+(E-$ $V(z)] / E_{\mathrm{g}}(z)$. Outside the barrier $V(z)=0$, hence $m_{0}(E)=m_{0}^{*}\left(1+E / E_{\mathrm{g}}^{0}\right)=$ $m_{0}^{*} \alpha_{0}$, where $E_{\mathrm{g}}^{0}$ is the energy gap of a semiconductor outside the barrier.

After somewhat lengthy but simple manipulations (as a guideline see for example derivation in [13]), we finally get the relationship between group time, delay time, self-interference time, and an additional nonparabolic term

$$
\tau_{\mathrm{g}}=\tau_{\mathrm{d}}+\tau_{\mathrm{i}}^{\mathrm{P}} / \alpha_{0}-\frac{\hbar}{2 k \mu_{0}} \int_{0}^{L} \frac{\partial \mu_{\mathrm{B}}}{\partial E} \frac{\partial \psi}{\partial z} \frac{\partial \psi^{*}}{\partial z} \mathrm{~d} z=\tau_{\mathrm{d}}+\tau_{\mathrm{i}}^{\mathrm{P}} / \alpha_{0}+\tau_{\mathrm{np}},
$$

with interference time in the case of parabolic dependence of energy on effective 
mass defined as $\tau_{\mathrm{i}}^{\mathrm{P}}=-\hbar \operatorname{Im}(R) / k(\partial k / \partial E)$, and $\mu_{\mathrm{B}}(z, E)=\hbar^{2} / m_{\mathrm{B}}(z, E)$ is reciprocal of effective mass within the barrier. It is easy to check that this reduces to the well known relation between group, delay and self-interference time if the nonparabolicity is absent, because the third term disappears and (3) then reduces to $\tau_{\mathrm{g}}=\tau_{\mathrm{d}}+\tau_{\mathrm{i}}^{\mathrm{P}}$.

\subsection{Real rectangular barrier}

Now we specialize to the text-book example of a rectangular barrier $(V(z)=$ $\left.V_{0}\right)$, where a fully analytic calculation is possible. The effective masses outside and inside the barrier are given by: $m_{0}(E)=m_{0}^{*}\left(1+E / E_{\mathrm{g}}^{0}\right)=m_{0}^{*} \alpha_{0}$ and $m_{\mathrm{B}}(E)=m_{\mathrm{B}}^{*}\left[1+\left(E-V_{0}\right) / E_{\mathrm{g}}^{\mathrm{B}}\right]=m_{\mathrm{B}}^{*} \alpha_{\mathrm{B}}$, where $m_{0}^{*}, m_{\mathrm{B}}^{*}, E_{\mathrm{g}}^{0}$, and $E_{\mathrm{g}}^{\mathrm{B}}$ are the position dependent effective masses and energy gaps outside and inside the potential barrier, respectively. The wave function inside the barrier is of the form $\psi_{\mathrm{B}}=A \mathrm{e}^{\kappa z}+B \mathrm{e}^{-\kappa z}$ where $\kappa^{2}=2 m_{\mathrm{B}}(E) / \hbar^{2}\left(V_{0}-E\right)$. From the continuity of the wave function and of $1 / m(z, E)(\mathrm{d} \psi / \mathrm{d} z)$, we can calculate the coefficients $A, B$, $T, R$, and consequently the relevant tunneling times. Here we give expression for the nonparabolic term only

$$
\tau_{\mathrm{np}}=\frac{\hbar}{2\left(E-V_{0}\right)} \frac{\alpha_{\mathrm{B}}-1}{\alpha_{\mathrm{B}}}\left(\frac{1+\delta^{2}}{4 \delta|g|^{2}} \sinh (2 \kappa L)-\frac{\Delta}{|g|^{2}} \kappa L\right) .
$$

where $\delta=m_{\mathrm{B}}(E) / m_{0}(E) \cdot(k / \kappa), \Delta=\left(1-\delta^{2}\right) /(2 \delta), g=\cosh (\kappa L)+\Delta \sinh (\kappa L)$, $\phi_{0}=-\arctan (\Delta \tanh (\kappa L)), \phi_{\mathrm{r}}=-\pi / 2-\arctan [\Delta \tanh (\kappa L)]$.

The tunneling times in the energy-independent effective mass case are easily obtained by setting $\alpha_{0}=\alpha_{\mathrm{B}}=1$, which is the case of coordinate dependent mass only. In this case we get the well known relation [13]: $\tau_{\mathrm{g}}=\tau_{\mathrm{d}}+\tau_{\mathrm{i}}$.

Suitable candidates for demonstrating the influence of nonparabolicity on tunneling times are material systems having substantial barrier heights, compared to the energy gaps in the materials, one of which is InGaAs/AlAsSb system. In

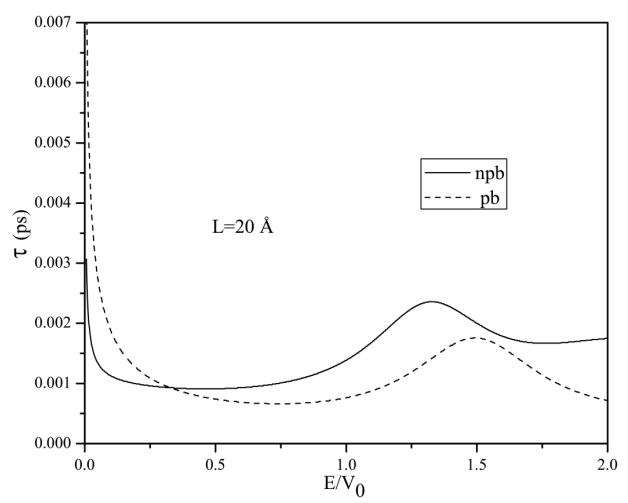

Fig. 1. The dependence of group time on the incident electron energy, calculated for a $\mathrm{In}_{0.53} \mathrm{Ga}_{0.47} \mathrm{As} / \mathrm{AlAs}_{0.56} \mathrm{Sb}_{0.44} / \mathrm{In}_{0.53} \mathrm{Ga}_{0.47}$ As structure with $L=20 \AA$ wide barrier layer, with (solid) and without (dashed line) the semiconductor nonparabolicity taken into account. 
particular, the calculated group time dependence on the particle energy in Fig. 1 confirms such expectations. It is worth noting that the inclusion of nonparabolicity is most significant at low particle energies.

\section{Complex potential}

We start with one-dimensional complex potential of the form $U_{\mathrm{c}}(z)=$ $U_{\mathrm{r}}(z)-\mathrm{i} U_{\mathrm{i}}(z)$. We shall suppose that both real and imaginary part of the potential occupy the same part $0<z<L$ of $z$ axis $(U(z)=0$ for all $z$ out of $(0, L))$. Further, we shall consider effective mass to be independent of both coordinate and energy throughout the structure.

Introduction of negative-definite imaginary part of the potential $\left(U_{\mathrm{i}}>0\right)$ describes absorption of the probability flux of the electronic system into unknown channels. Absorption $\mathcal{A}$ is given by

$$
\mathcal{A}(E)=\frac{2}{\hbar} \int_{0}^{L} U_{\mathrm{i}}|\psi(z)|^{2} \mathrm{~d} z / J_{\text {inc }}=\frac{2 m}{\hbar^{2}} \int_{0}^{L} U_{\mathrm{i}}|\psi(z)|^{2} \mathrm{~d} z
$$

where $J_{\text {inc }}=\hbar k / m$ is the incident particle flux, impinging on the barrier from $-\infty$.

Using the definition of the current and the expressions for the wave function in front of and behind the barrier (the same form as in (2) only with complex wave number), a quasi-unitary condition is obtained: $\mathcal{R}(E)+\mathcal{T}(E)+\mathcal{A}(E)=1$, where $\mathcal{T}=|T|^{2}$ and $\mathcal{R}=|R|^{2}$ are transmission and reflection probabilities.

We employ the same procedure as in the first section to get the relationship between the tunneling times, and give only the final result

$$
\tau_{\mathrm{d}}=\tau_{\mathrm{g}}-\tau_{\mathrm{i}}-\frac{2 m}{\hbar k} \int_{0}^{L} U_{\mathrm{i}} \operatorname{Im}\left(\psi^{*} \frac{\mathrm{d} \psi}{\mathrm{d} E}\right) \mathrm{d} z=\tau_{\mathrm{g}}-\tau_{\mathrm{i}}+\tau_{\mathrm{a}},
$$

where $\tau_{\mathrm{a}}$ originates from the finite value of the imaginary part of the potential. In the absence of the absorption $\left(\tau_{\mathrm{a}}=0\right)$, Eq. (6) directly reduces to the well known relationship [13]: $\tau_{\mathrm{d}}=\tau_{\mathrm{g}}-\tau_{\mathrm{i}}$.

\subsection{Rectangular complex barrier}

Now we turn our attention to the case of a complex potential extending over the finite region of space. We choose a simple convenient one-dimensional rectangular-like potential [15]: $U=\left[\hbar^{2} /(m L)\right]\left(\beta_{\mathrm{r}}-\mathrm{i} \beta_{\mathrm{i}}\right), 0<z<L$, and $U(z)=0$ otherwise, with $\beta_{\mathrm{r}}$ and $\beta_{\mathrm{i}}$ being positive constants.

We search for the potential configuration leading to the maximum reduction of the group time $\left(\tau_{\mathrm{g}}=\tau_{\mathrm{d}}+\tau_{\mathrm{i}}-\tau_{\mathrm{a}}\right)$. The following dimensionless parameters are defined as $K=k L, B_{\mathrm{r}}=2 \beta_{\mathrm{r}} L$ and $B_{\mathrm{i}}=2 \beta_{\mathrm{i}} L, k$ being the wave number outside complex barrier. Two limiting cases are of prime interest: the long wavelength limit, which corresponds to $K \ll 1$ or $\lambda=2 \pi / k \gg L$, and the short wavelength limit $K \gg 1$ or $\lambda \ll L$.

The large values of $\tau_{\mathrm{a}}$ in the long wavelength limit $K \ll 1$ are obtained for predominantly imaginary potential (see Fig. 2). Increase in $K$ shifts away 


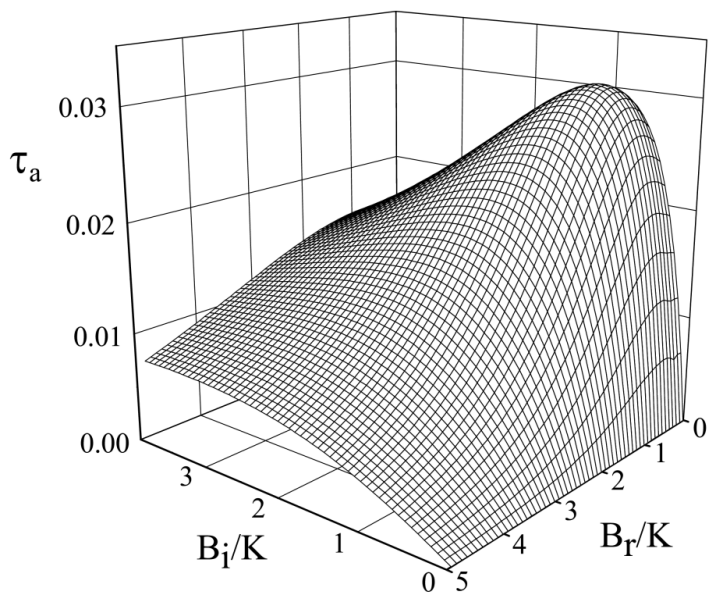

Fig. 2. Dependence of $\tau_{\mathrm{a}}$ (relative units as in Fig. 1) on real and complex parts of the potential for $K=0.2$ (long wavelength case). For this potential configuration the incident particle flux is distributed as follows: $\mathcal{T}=0.43, \mathcal{R}=0.13, \mathcal{A}=0.44$.

the absolute maximum from purely imaginary potentials (Fig. 2). In the short wavelength regime $(K \gg 1)$ we get the maximum values of $\tau_{\mathrm{a}}$ for the imaginary part of the potential negligible compared to the real part (the maximum absorption $(\mathcal{A}=1)$ in the short wavelength limit is reached for purely imaginary potential).

\section{Conclusion}

Tunneling of a particle with energy dependent effective mass through onedimensional potential barrier was considered, and general relations between phase, group, and dwell times are derived for an arbitrary potential. Depending on the material parameters, i.e, barrier height, energy gap values etc., accounting for the nonparabolicity may increase the group time up to $30 \%$ in realistic structures.

Also, for the case of complex potentials we extend present theory to account for the absorptive tunneling time $\tau_{\mathrm{a}}$. Its influence on group delay is most pronounced for mainly imaginary potential in the long wavelength limit. Contrary to it, in the short wave length limit the maximum reduction in the group time is found for the potentials with only small imaginary part.

\section{References}

[1] F. Capasso, K. Mohammed, A.Y. Cho, IEEE J. Quantum Electron. QE-22, 1853 (1986)

[2] Y. Guo, C.E. Shang, X.Y. Che, Phys. Rev. B 72, 045356 (2005).

[3] D. Bohm, Quantum Theory, Prentice-Hall, New York 1951.

[4] E.P. Wigner, Phys. Rev. 98, 145 (1955).

[5] M. Büttiker, Phys. Rev. B 27, 6178 (1983). 
[6] J.G. Muga, Phys. Lett. A 167, 24 (1992).

[7] W. Li, Y. Guo, Phys. Rev. B 73, 265311 (2006).

[8] H.G. Winful, M. Ngom, N.M. Litchnitsev, Phys. Rev. A 70, 052112 (2004).

[9] H.M. Nussenzveig, Phys. Rev. A 62, 042107 (2000).

[10] E.H. Hauge, J.A. Støvneng, Rev. Mod. Phys. 61, 917 (1989).

[11] C.R. Leavens, G.C. Aers, Phys. Rev. B 39, 1202 (1989).

[12] S. Brouard, R. Sala, J.G. Muga, Phys. Rev. A 49, 4312 (1994).

[13] H.G. Winful, Phys. Rev. Lett. 91, 260401 (2003).

[14] F.T. Smith, Phys. Rev. 118, 145 (1960).

[15] P. Molinas-Mata, P. Molinas-Mata, Phys. Rev. A 54, 2060 (1996). 